\title{
evoDash - A Transdisciplinary Vision for an Education Platform and a Simulation- Based Vehicle Development Process
}

\author{
Lucas KUENTZER ${ }^{1}$, Marcel SCHWARZENBARTH, Daniel SILADJEV and Georg \\ ROCK $^{2}$ \\ Trier University of Applied Sciences
}

\begin{abstract}
In the wake of environmental disasters and accelerating climate change the challenges facing humanity seem bigger than ever. In the public eye private transport and mobility are two of the most apparent fields in need of a sustainable evolution. Around the globe car manufacturers and developers of innovative mobility solutions are hard at work in shaping the future of transport and travel. Like many modern problems these fields require a transdisciplinary approach and collaboration of disciplines in order to design a solution. At Trier University of Applied Sciences, the student team proTRon has been building highly efficient mobility concepts since 2005 and developing the prototype for a law- and safetycompliant urban vehicle concept since 2015. In this industry-oriented collaboration project the students get the chance to work in a realistic environment emulating a vehicle development process, preparing them for a job in the mobility industry as the next generation of system developers and engineers with a transdisciplinary attitude. Within the framework of this project students acquire competencies in communication and cooperation as well as gain expertise in areas like sustainability, efficiency, and organization. This paper introduces "evoDash", a human-vehicle interface prototype for the urban vehicle concept proTRon EVOLUTION with a focus on usability and modularity. Designed and developed by students it is a software architecture based on Android and central part of a vision for a transdisciplinary education platform, which provides the foundation for future software and hardware development projects working towards an innovative and sustainable human-vehicle interface. The modular architecture of the platform provides the necessary interfaces and layout options for the functionalities that result from innovative ideas and student projects, embedding them into a usable and individually adjustable framework that will be subject to continuous iterations in order to optimize usability, safety and security. This paper proposes a simulationbased process model focused on rapid prototyping. It aims at providing a possible framework for transdisciplinary engineering projects and education.
\end{abstract}

Keywords. transdisciplinary, sustainability, usability, human-vehicle interface, human-centered design, higher education

\footnotetext{
${ }^{1}$ Corresponding Author, Mail: L.Kuentzer@hochschule-trier.de.

${ }^{2}$ Corresponding Author, Mail: G.Rock@hochschule-trier.de.
} 


\section{Introduction}

Global challenges like climate change are further facilitated by an emerging world and those problems are becoming more and more complex and all-encompassing, so that simple multi- and interdisciplinary collaboration are not enough to deal with them anymore. Fields like transport and mobility in the light of sustainability as a guiding principle deal with problems and propose solutions that have a major impact on the society, the economy and the environment. Researchers and experts from social-sciences, economics, natural sciences and technical disciplines must work together in a forum of open communication using methodologies and tools that go beyond their original fields to come up with the solution to modern problems [1].

At Trier University of Applied Sciences, the student team proTRon has been developing highly energy-efficient vehicle prototypes since 2005 and shifting their attention to the development of a street-legal, urban vehicle, that is supposed to challenge the established understanding of individual mobility in the automotive industry by advocating that less is more [2]. Starting as an extra-curricular activity, it has developed into a course-credited, but student-driven project with high ambitions. In collaboration with various departments, students with a background in electrical and automotive engineering, computer science, economics or design can contribute to this vision of a sustainable future of mobility in an industry-oriented study environment.

In this context higher education has the freedom to be a playing field for bright ideas without the constraints of economic risk. Students are free to pursue creative and innovative ideas, while retaining the option to fail and learn from it. There is a certain advantage in not having to play it safe. As a result, this paper proposes a partial solution to the challenges of the changing automotive industry in the form of a contemporary human-vehicle interface prototype and ambitious visions for a transdisciplinary education platform and a simulation-based development process.

\section{Transdisciplinary Innovation}

Transdisciplinarity commonly describes a research strategy adhering to the idea of a unified truth or shared knowledge regarding the workings of the world, transcending the boundaries of separated disciplines and building on top of them [1]. Perhaps a more comprehensible understanding in the scope of practice-oriented engineering projects is that of high-level integration of methodology and processes of multiple - if not all disciplines [1].

Modern technological advancements and solutions cross the boundaries of disciplines. A transdisciplinary approach involves open communication between all imaginable stakeholders and taking into consideration current state, goals and outcomes in the grand scheme of scientific, social and ecological interaction [1].

Such an approach is especially necessary in the context of sustainable development and manufacturing. Sustainability as the concept of future-proof planning and preservation is inherently a transdisciplinary field, due to the natural interactions between systems, manmade or not [3]. 


\subsection{Transdisciplinary Engineering in Vehicle Development}

In the past the development of a car mainly represented the organizational structures of the automotive companies and suppliers themselves [4]. Each department developed their domain-specific solution for a problem, with department A for example developing the engine control unit, while department B was responsible for the body control unit. Thus, functions were designed locally and isolated [4,5].

Bus systems were introduced to enhance wiring and ECUs, sensors and actuators were interconnected for the first time, enabling new distributed functionality, such as ESP or ABS [4,5]. Consequently, even the development of the electrical system of a vehicle became a highly multidisciplinary task combining the knowledge of mechanical, electrical, electronical and software engineers. In order to develop such vehicle functions it is essential that these people have the same understanding of the problem, processes and solution $[6,7]$.

Already today, modern vehicles consist of several million lines of code and include up to hundred electrical control units. Due to the development towards connected and autonomous vehicles and the software being the major driver for innovations, the amount of software in the car is predicted to rise even more drastically [8,9]. Next to brand, exterior design and energy efficiency of the vehicle, build-in software is increasingly turning into a deciding factor in the customer's vehicle preference [9]. Therefore, automotive manufacturers have increasingly started to treat in-car software as yet another important distinctive feature of their vehicles. One main component in this is the humanvehicle interface. Car manufacturers have the opportunity to create a diverse, distinctive and on-brand way of interaction for their vehicles [9].

Besides the increasing introduction of software in the car, the amount of information technology in the automotive industry, such as through off-board computing, cloud computing, connected and shared mobility services will also increase [9].

Because of the increasing amount of such interconnected, distributed softwareenabled functions the complexity is growing dramatically, which transforms it to an organizational challenge for the automotive industry to handle. Challenges such as autonomous driving, connected cars, electrical and shared mobility for a more sustainable, safer and comfortable driving pose complex problems. The automotive industry is currently facing the reality, that their established methods and structures are not sufficient anymore to solve these problems [6]. In order to develop ethical solutions, aspects like the legal position and urban planning have to be considered. The development process does not only involve car manufacturers, but also the government, the infrastructure and the society, making it a highly transdisciplinary field [6].

These challenges will continuously require established processes and methodologies of the automotive industry to change [8]. Most manufacturers currently do not have the in-house expertise to stay on top of these developments and are even more dependent on various suppliers in order to provide elevated customer experiences, find sustainable solutions for future mobility and reduce costs [8]. Therefore, they cooperate with IT companies or other car manufacturers to get the required know-how and combine resources in order to master these trends [10]. At the same time big OEMs are building up their own expertise in information technology through launching their own IT development centers, as to not risk becoming a mere platform supplier for the humanvehicle solutions of IT giants [10].

Even titans and rivals of the automotive industry like Daimler and BMW are joining forces on research and development for a more sustainable future mobility with shared 
platforms such as Charge Now, Park Now or the car sharing service Share Now [11]. The changing industry introduces challenging tasks, but also a high potential for new innovations.

\subsection{Transdisciplinary Education}

More than integrating disciplines and fusing methodologies, Transdisciplinary Education places a focus on promoting transdisciplinarity as a meta-discipline and as an open, scientific way of thinking, which takes surrounding systems and the shared knowledge between and above disciplines into consideration [1]. The goals of Transdisciplinary Education are to develop a standard as to what a transdisciplinary approach entails and to providing established measures for the transdisciplinary competencies of students [1].

Unfortunately, the pursuit of transdisciplinarity can be hard within the confines of the fragmented structure of higher education and academics. The education institutions are organized into departments, often competing with one another for resources. Students are expected to pick a field to specialize in and are hardly ever incentivized by the curriculum to think outside the box [12]. Even in multi- or interdisciplinary collaborations students are limited by the discipline-specific methodology taught in their monodisciplinary curricula [1]. This inhibition of a transdisciplinary evolution peaks with the discipline-specific structure of academic journals [1].

At the same time, student learning is changing as they are studying more individually based and at their own pace. The role of teachers in higher education is shifting from being the performing agent to providing the resources, background and environment for students and their individual study [3]. Students are more and more interested in learning how to work sustainably and research suggest that students benefit immensely from coupling teaching sustainability and transdisciplinarity, as they are naturally related [3].

The intertwined relation of the two concepts is apparent in education but can be observed in application as well [3]. Students need to be provided with a learning environment and context that facilitates their transdisciplinary development. Major changes in established and traditional structures are unlikely to be accepted, as they threaten the status quo [1]. Instead institutions are to be encouraged to pursue establishment of transdisciplinary competencies through workshops and department collaboration projects $[1,3,12]$.

\section{2. evoDash - a Concept for an innovative Human-Vehicle Interface}

The student-centered vehicle development project "proTRon EVOLUTION" is such a collaboration project capable of teaching transdisciplinary competencies. The goal of the project is the development of a street legal and highly efficient, electric urban vehicle in the name of sustainable mobility. "evoDash" is a sub-project, currently helmed by IT students and originally intended as a regular display-based infotainment system, which has become an industry standard [13]. Now, the "evoDash" prototype sits at the center of our vision for a modern and innovative human-vehicle interface, that goes beyond entertainment, navigation and basic driving data. The main focus of both the project and the sub-project is sustainability and efficiency not only during usage of the finished product, but also in development and production. The industry standard for infotainment systems is big, heavy, overprized and resource intensive hardware that remains unused while the vehicle is not being operated. A more efficient approach is to move the 
processing power out of the vehicle where possible and into the driver's mobile devices. Those are often cheaper, lighter and have use outside the vehicle. By making the processing unit independent of the physical vehicle, upgrading the hardware becomes easier as the user can interchange mobile devices for linking up to the vehicle to stay upto-date with developments in hard- and software. Because the development cycle of about three to four years for a car is much longer than the advancements in information technology, the car could be equipped with state-of-the-art technology even after start of production [7]. As a consequence, the lifecycle period of modern vehicles could be increased and value retained.

Obviously, redundancies, safety and security concerns need to be considered, but such an approach would considerably increase the sustainability and eco-friendliness of a new generation of vehicles. Furthermore, "evoDash" (ref. Figure 1) has become a vision for a human-vehicle interface focused on interaction, sustainability and individuality. The system could encourage resource-friendly driving by impacting behavior through optimization, gamification and emotion to train the driver and raise awareness for sustainable driving. Machine learning can play a role in optimization depending on driver, vehicle, route and taking into consideration vehicle attributes like recuperation, route details like height profiles and traffic, and driver goals like commute and a fun driving experience.

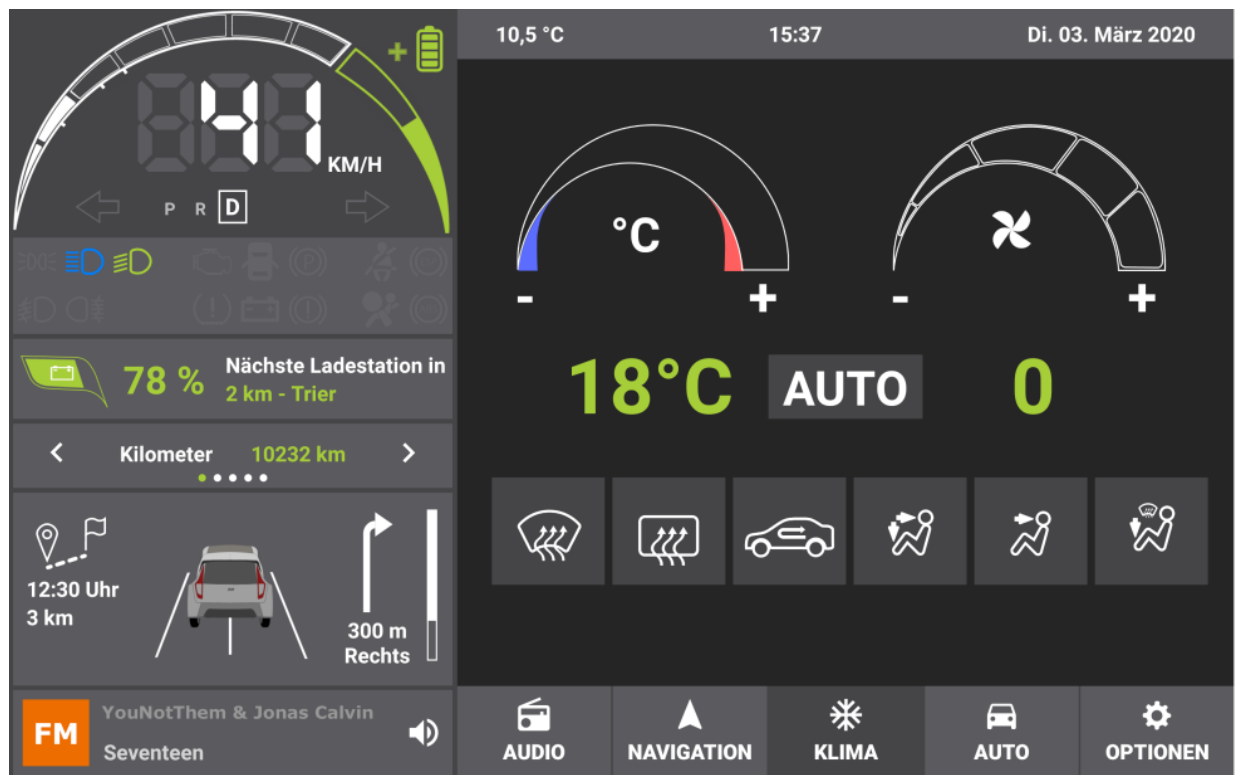

Figure 1. Display-based UI of the human-vehicle interface Android prototype "evoDash" with the climate control screen currently displayed on the right and dynamic driving data displayed on the left.

The application prototype itself was developed with the goals of high modularity, adaptability and individuality. As outlined above, the whole system is supposed to improve vehicle attractiveness, especially for a sustainable and low-comfort vehicle like the concept being developed by the proTRon team. 


\subsection{System Development Process for a Human-Vehicle Interface Application}

When developing a human-vehicle interface various legal, safety and security obligations have to be considered. Accordingly, applications like "evoDash" have to be compliant with German Road Traffic Licensing Regulations, with the current speed being displayed at all times just being one of many stipulations. Additionally, it is important that the driver does not get distracted while operating the car and that the user interface remains unobtrusive [14].

As such, development of digital human-vehicle interface applications, such as the "evoDash" prototype, is a highly transdisciplinary task. Not only is the necessary skill set and expertise of multiple disciplines required for the development, but the whole system needs to be considered for every stakeholder. To ensure that the intended user benefit from the system and find it appealing, quality features like usability, performance, reliability and overall aesthetics need to be validated through an open forum made up of experts from multiple backgrounds [15].
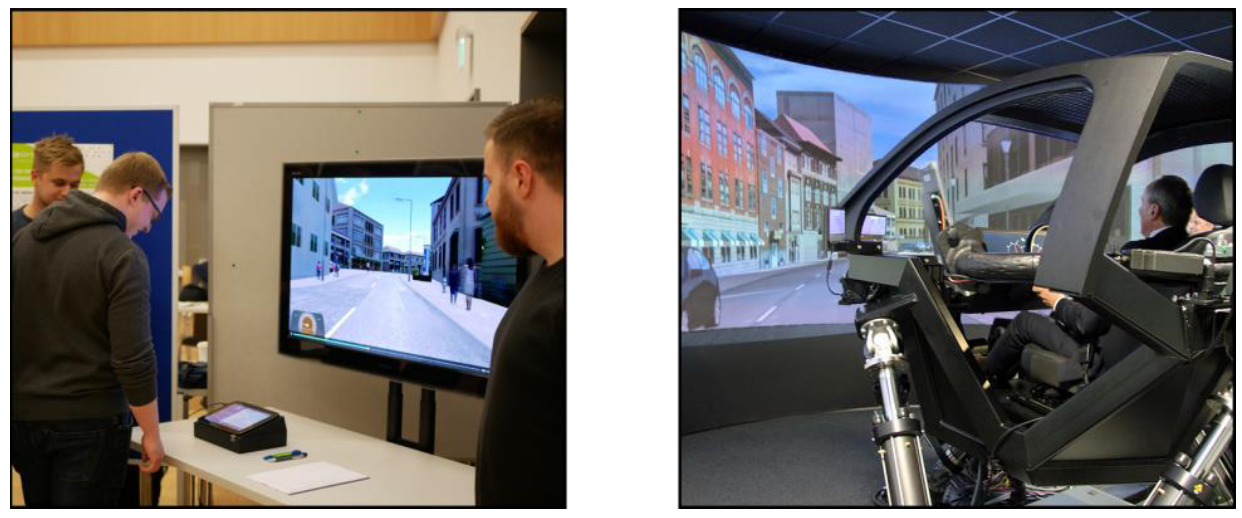

Figure 2. "evoDash" demo on an Android tablet during a "rolling chassis" event on the left and the driving simulator "FaSiMo" during a test drive on the right.

There are numerous psychological and cognitive aspects of humans interacting with a system interface in a safety-critical environment, that also must be considered. The driver should be able to perceive information of the user interface easily and navigate through the system quickly and accurately to reduce driver distraction [14].

The key to a transdisciplinary development like this, is that the different stakeholders involved in the process are equally informed of the development status and have the same understanding of the system [6]. For "evoDash" graphical mock-ups and use-case diagrams and other design artifacts were created to evaluate and validate different usability and application scenarios among the stakeholders of the project to prevent misunderstandings and identify problems long before the first line of code was written $[13,16]$.

Due to the fact that the physical test vehicle of the "proTRon EVOLUTION" is not ready to be put on the road, the driving simulator (ref. Fig. 2) at the University of Applied Science in Trier was used to test the current iteration of the "evoDash"-prototype with driving data from a simulated test track [17]. "evoDash" has been part of several events (ref. Fig. 2) at the University of Applied Science in Trier, where visitors had the opportunity to experience this human-vehicle interface prototype and provide feedback 
regarding its usability, which could then be implemented in the next iteration of the modular and adaptable application architecture as further outlined in section 2.3.

\subsection{User Interface Guidelines for Vehicle Development}

With respect to consistency, usability and accessibility, user interface guidelines for vehicle development were introduced into the process of creating the "evoDash" prototype. This approach was obligatory to be able to construct a quickly adaptable user interface. The challenge is to provide driving information and entertainment data in an efficient way so that users rapidly figure out how to operate the application.

These guidelines are not intended as rules or restrictions but rather as convenient recommendations to support the procedure of turning goals and requirements into an applicable design [18]. General design guidelines which are already established in modern mobile devices are often too abstract or not befitting the concept [18]. Even though these existing design guidelines generally differ in their intention and output, in our case they are suited as a structure that can be adapted to draft design guidelines, tailored to the system $[13,19]$. In the development of an application for a street-legal vehicle it is crucial to take passengers' safety into account.

One focus of our approach in the development of our user interface guidelines are the ten heuristics by Molich and Nielsen [20]. They provide a classification of usability problems, that influenced the user interface guidelines of the „evoDash“ user interface. These classifications are quite similar to Google's Material Design [21]. Material Design describes the utilization of physical rules and flat surfaces to imitate materials that help the user indicate areas where user interaction is possible. The use of very graphical and bold elements guides the user through the learning process on how to interact with the given components. The simplicity of flat design has the function to enhance interactions with the system. Important information visually pops to draw the attention of the user [21].

Overall, the „evoDash“ user interface guideline helped with building a consistent and user-friendly interface design that assists the driver in effectively interacting with the UI, while still adhering to the traffic regularities.

\subsection{Transdisciplinary Education Platform}

The student project "proTRon EVOLUTION" as a vehicle development project already unites many different disciplines in its vision of a light-weight, high-efficiency electric vehicle for private or shared mobility in urban areas. Contrary to the direction of the German automotive industry, which currently aims for luxury and comfort, the vehicle concept focuses on the "mobility" keystone: safe, reliable and affordable travel. This means sacrificing the comfort and luxury of the physical driving experience, making it even more crucial to add value and attractiveness through software and clever concepts.

Currently the development is mostly multidisciplinary in nature with students largely following methodologies taught in the curriculums of their respective departments. However, there are some sub-projects, such as the development of the „evoDash“ infotainment concept that follow the transdisciplinary idea. After the completion of the test vehicle, automotive engineering as the driving force behind the project will only be able to generate little additional value through optimization. 
Instead the focus of the project will shift towards electrical engineering and information technology and the generation of value through innovative software functions. In the context of driver experience design and vehicle performance optimization various disciplines like economics, social studies and environmental sciences play a part and while their perspectives are to be considered, the collaboration project "proTRon" is supported most strongly by the technical faculties. The shift towards software as the primary value generator for the product is an opportunity to create a transdisciplinary framework for an education platform, a pilot project for transdisciplinary work and the prototype for a vision of modern human-vehicle interaction. The focus of the overall project will then shift towards usability, individuality, data security, adaptive performance and energy optimization, vehicle information, driver interaction and attractiveness of the overall driving experience. All these issues have to be approached with a transdisciplinary mindset due to their multilateral nature, requiring student teams of different disciplines.

Innovative development, cooperation and competition with the automotive industry and practical education of the students for their future in the workforce remain the cornerstones of the project. It provides the students with an environment to develop skills in solving problems independently, responsibly, and sustainably, while being able to communicate and collaborate on them. Students are supposed to get the chance to try out new methods and pursue innovative ideas in a framework that promotes crossing the boundaries of the disciplines.

„evoDash”, which was initially only envisioned as a regular infotainment system and dashboard display has evolved into a vision for modern human-vehicle interaction based on usability, individuality, emotion and clarity. In order to carry such a transdisciplinary vision into multi- and interdisciplinary working groups made up of students of different departments, the established methodologies and development models of the departments will not be sufficient. Due to that this paper proposes a novel process model, fit for vehicle development and its context, in the next section.

\section{Transdisciplinary Development Model for simulation-based Prototyping}

In order to develop a safe, functional and usable human-vehicle interface, design a fulfilling driving experience and optimize performance and efficiency per individual, multidisciplinary student teams in project "proTRon" require methods beyond the ones taught in the curriculum of their departments. Figure 3 proposes a process model that takes into consideration the transdisciplinarity that the project vision requires. Initially limited to mainly contributing disciplines, the model can be expanded upon contributors.

Several contextual factors, such as costs, safety and fragmented development cause the simulation-based nature of this prototype development model, fit around the development of the "proTRon EVOLUTION". Students from information technology, automotive and electrical engineering work together in tandem to translate the shared, common knowledge and innovative vision into discipline-specific components of an interactive system. The inner loop iterates in a simulation environment before deploying into the outer loop and onto the physical test vehicle as a prototyping platform. There, the system gets validated and updated requirements lead back in an agile fashion into the inner loop for the optimization of interaction, usability and sustainability. Eventually the first iteration of a finished vehicle can be deployed, while iterative development continues. 
Obvious use-cases for this approach include the implementation of machine learning for adaptable optimization of range, energy-consumption and performance based on the individual vehicle, driver, or route. It also provides a framework for fine-tuning driving experience and human-vehicle interaction. There is an opportunity to develop a system that influences the driver's behavior towards sustainable driving based on the sensory self-perception of the vehicle and the dynamic and context-based relay of information.

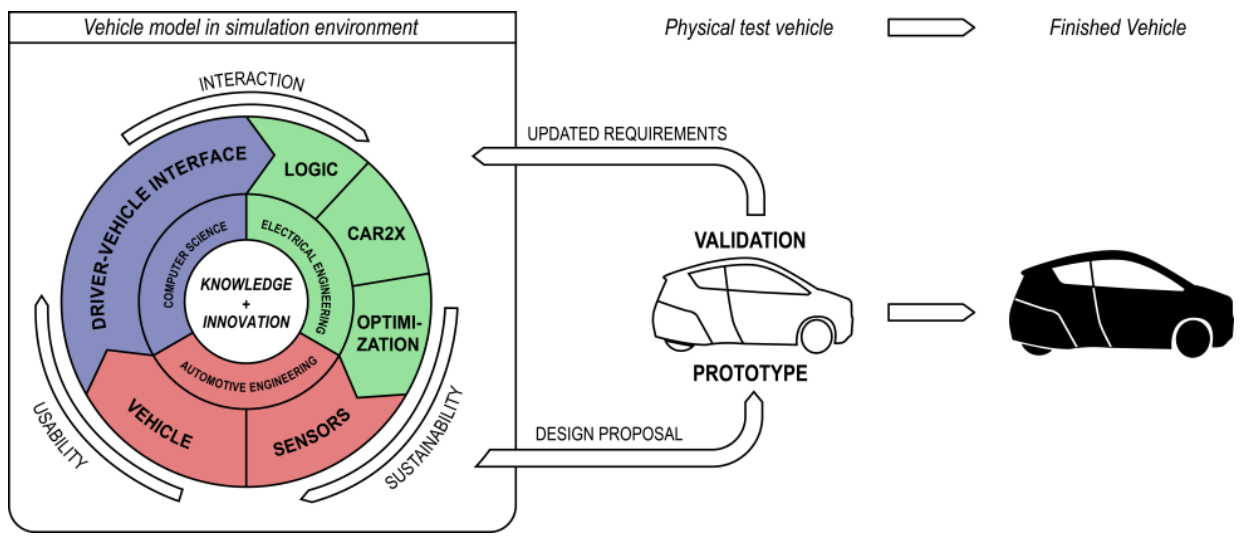

Figure 3. Process diagram of a simulation-based prototype development model in a transdisciplinary project environment with multiple departments collaborating.

\section{Conclusion}

"evoDash" is a promising vision for modern human-vehicle interaction, especially in the context of sustainability. At Trier University of Applied Sciences, it is part of a platform that encourages students to develop transdisciplinary competencies through multi- and interdisciplinary teamwork and collaboration towards a common goal. The paper presents a student project, which aims for competitive innovation without being tied to the typical industrial framework. The freedom gained in the development of open solutions shows how the described transdisciplinary approach can be successfully implemented. The procedure model presented combines agility with prototypical quality assurance without restricting its flexibility.

Just recently established as a project platform, "evoDash" is already providing a framework for several student projects in the IT department. Those projects focus on the link between the vehicle systems and the user interface, such as radio or context-driven navigation and subsequently cross over into fields such as automotive and electrical engineering. Only time will tell if "evoDash" proves successful in its educational goals, but based on the results and environment the "proTRon" project provides on the engineering side, we are confident in the benefit "evoDash" provides for our students.

The conceptual ideas and innovations regarding "evoDash" must also pass the proof of time. Nevertheless we believe in this respect that established car manufacturers will run into problems in the future with the wide variety of proprietary user interaction hardware/software and will have to take other paths, perhaps similar to the one pursued here. 


\section{Acknowledgement}

Sincere thanks to all the members of the projects "proTRon" and "FaSiMo" at Trier University of Applied Sciences for their support and feedback. Special thanks also to the co-authors of "evoDash": Jonas Reetz, Sascha Reinshagen and Damir Zinn.

\section{References}

[1] V. Mokiy, International Standard of Transdisciplinary Education and Transdisciplinary Competence, Informing Science: the International Journal of an Emerging Transdiscipline, vol. 22, 2019, pp. 73-90.

[2] Hochschule Trier, 2020, proTRon, Accessed: 15.02.2020. [Online]. Available: https:/hochschuletrier.de/go/protron

[3] D. Gillis et al., Interdisciplinary and Transdisciplinary Research and Education in Canada: A Review and Suggested Framework, Collected Essays on Learning and Teaching, vol. 10, 2017, pp. 203-222.

[4] W. Zimmermann and R. Schmidgall, Bussysteme in der Fahrzeugtechnik: Protokolle, Standards und Softwarearchitektur, Springer Vieweg, $5^{\text {th }}$ ed, Wiesbaden, 2014.

[5] M. Broy, Challenges in Automotive Software Engineering, In: ICSE'06: Proceedings of the 28th International Conference on Software Engineering, Shanghai, 2006, pp. 33-42.

[6] S. Maro, The Automotive Domain-From Multi-Disciplinarity to Trans-Disciplinarity, Proceedings, vol. 1, 2017, pp. 172-175.

[7] J. Schäuffele and T. Zurawka, Automotive Software Engineering: Grundlagen, Prozesse, Methoden und Werkzeuge effizient einsetzen, Springer Vieweg, $6^{\text {th }}$ ed, Wiesbaden, 2016.

[8] C. Ebert and J. Favaro, Automotive Software, IEEE Software, vol. 34, 2017, pp. 33-39.

[9] O. Burkacky, J. Deichmann, G. Doll and C. Knochenhauer, 2018, Rethinking car software and electronics architecture, Accessed: 20.02.2020. [Online]. Available: https:/www.mckinsey.com/ industries/automotive-and-assembly/our-insights/rethinking-car-software-and-electronics-architecture

[10] Volkswagen AG, 2019, Mobilität für kommende Generationen. Geschäftsbericht 2019, Accessed: 15.05.2020. [Online]. Available: https://www.volkswagenag.com/presence/investorrelation/ publications/annual-reports/2020/volkswagen/Y_2019_d.pdf

[11] Daimler Mobility AG, 2020, BMW und Daimler bündeln Mobilitätsdienste, Accessed: 18.02.2020. [Online]. Available: https:/www.daimler-mobility.com/de/unternehmen/news/joint-venture-closing/

[12] J. Merck and M. Beermann, The Relevance of Transdisciplinary Teaching and Learning for the Successful Integration of Sustainability Issues into Higher Education Development, In W. L. Filho et al. (eds.): Integrative Approaches to Sustainable Development at University Level, Springer International Publishing, Switzerland, 2015, pp. 19-25.

[13] M. Schwarzenbarth, D. Siladjev, J. Reetz, S. Reinshagen and D. Zinn, Entwicklung eines Frameworks für ein digitales User Interface zur Anzeige von Fahrzeug- und Infotainmentdaten für den proTRon EVOLUTION, Department of Computer Science, Trier University of Applied Sciences, 2020.

[14] S. Geisler, Menschliche Aspekte bei der Entwicklung von Fahrassistenzsystemen, In C. Reuter: Sicherheitskritische Mensch-Computer-Interaktion: Interaktive Technologien und Soziale Medien im Krisen- und Sicherheitsmanagement, Springer Vieweg, $1^{\text {st }}$ ed, Wiesbaden, 2018, pp. 337-356.

[15] F. Grandi, M. Peruzzini, L. Zanni, C. E. Campanella and M. Pellicciari, Digital Manufacturing and Virtual Reality for Tractors' Human-Centred Design, Advances in Transdisciplinary Engineering, Vol. 7, 2018, pp. 702-711.

[16] I. Sommerville, Software Engineering, Pearson Education Limited, 10 ${ }^{\text {th }}$ ed, Harlow, 2016.

[17] Hochschule Trier, 2020, Fahrsimulator, Accessed: 15.02.2020. [Online]. Available: https://hochschuletrier.de/go/fahrsimulator

[18] S. Cronholm, The Usability of Usability Guidelines - a Proposal for Meta-Guidelines, In: OZCHI '09: Proceedings of the 21st Annual Conference of the Australian Computer-Human Interaction Special Interest Group: Design: Open 24/7, Melbourne, 2009, pp. 233-240.

[19] E. Karana, B. Barati, V. Rognoli and A. Z van der Laan, Material Driven Design (MDD): A Method to Design for Material Experiences, International Journal of Design, vol. 9(2), 2015, pp. 35-54.

[20] R. Molich, J. Nielsen, Improving a Human-Computer Dialogue, Communications of the ACM, vol. 33(3), 1990, pp. 338-348.

[21] Google LLC, 2014, Introduction - Material Design, Accessed: 15.02.2020. [Online] Available: https://material.io/design/introduction/\#principles 